\title{
Optimisation de la section d'un canal de navigation Études par modèles mathématiques et physiques
}

\author{
Optimization of a canal cross-section \\ Mathematical and scale model studies
}

\author{
M. Pommier et M. Selmi \\ Compagnie Nationale du Rhône
}

Dans le cadre des études qui lui ont été confiées par le Ministère des transports en vue de la mise au point du projet de liaison navigable à grand gabarit, la Compagnie Nationale du Rhône a été amenée à rechercher l'influence de tous les paramètres qui régissent le déplacement des convois poussés le long d'une voie navigable en particulier le long des canaux artificiels ou des rivières ainsi qu'à l'entrée ou la sortie des écluses.

Cette recherche a été conduite à partir de calculs théoriques, permettant toutes les extrapolations désirées, appuyés sur les résultats d'essais physiques sur modèle réduit.

\section{Données de base}

En ce qui concerne de façon plus particulière les canaux de navigation, les données de base retenues sont les suivantes:

\section{Dimensions du convoi type}

- 1 pousseur de 30 mètres de longueur et de 11,40 mètres de largeur ;

- 1-2-3 barges de 76,50 mètres de longueur et de 11,40 mètres de largeur.

\section{Vitesse normale du convoi}

$-8,5 \mathrm{~km} / \mathrm{h}$ (soit $2,36 \mathrm{~m} / \mathrm{s}$ ) conformément aux indications de la circulaire du 1er Mars 1976 relative aux caractéristiques des voies navigables.

\section{Puissance du pousseur}

0,5 CV par tonne transportée pour l'enfoncement de

$3 \mathrm{~m}$. Tenu compte du rendement du système de propul- sion et pour un fonctionnement normal des moteurs à $60 \%$ de leurs possibilités, la puissance utile correspondante est de l'ordre de $350 \mathrm{CV}$.

\section{Marche des calculs et essais}

De simples considérations d'analyse dimensionnelle montrent que dans un canal, la résistance à l'avancement d'un bateau est, en principe, proportionnelle au carré de sa vitesse de déplacement par rapport à la berge augmentée de la vitesse du courant de retour.

Les calculs et essais ont donc porté successivement sur :

- le courant de retour en fonction des différents paramètres caractérisant le canal, le convoi et son déplacement ;

- la résistance opposée à l'avancement du convoi.

\section{Courant de retour}

\section{Etude théorique}

L'application du théorème des quantités de mouvement associé à l'équation de continuité permet de définir les relations qui lient l'abaissement du convoi et la vitesse du courant de retour à la vitesse du convoi.

Equation de continuité

Le canal étant supposé être en eau tranquille, cette équation exprime l'égalité entre le volume déplacé du bateau par unité de temps et le volume d'eau arrivant en sens inverse par le courant de retour. Cette équation s'écrit :

$$
v=\frac{s+L \Delta h}{s-s-L \Delta h} V
$$


où

$S=$ section mouillée du canal

$L=$ largeur du plan d'eau

$s=$ surface du maître couple du bateau

$V=$ vitesse du bateau

$v=$ vitesse du courant de retour

$\Delta h=$ abaissement du bateau

$v$ et $\Delta h$ peuvent être pris dans tout profil en travers et notamment au droit de la poupe.

\section{Théorème des quantités de mouvement}

Le débit de quantité de mouvement sortant d'une surface donnée est égale à la somme des forces extérieures agissant sur cette surface :

$$
\iint_{s} \rho \cdot V_{n} \cdot \vec{V} \cdot d s=\Sigma \overrightarrow{\text { forces extérieures }}
$$

\section{Variation de quantité de mouvement}

Le canal étant horizontal et la surface choisie étant constituée par un tronçon de canal limité à l'amont par une section droite du canal et à l'aval par une section droite à l'aplomb de la poupe, cette variation s'écrit :

$$
\rho\left[(V+v)^{2}\left(1+\frac{\alpha-1}{3}\right)(S-s-L \Delta h)-V^{2} S\right]
$$

dans laquelle :

$$
\begin{aligned}
1+\frac{\alpha-1}{3}= & \text { coefficient de quantité de mouvement } \\
\alpha= & \text { coefficient d'énergie cinétique ou de } \\
& \text { Coriolis qui a été pris égal à } 1,10 \\
\rho & =\text { masse volumique de l'eau }
\end{aligned}
$$

Dans l'établissement de cette relation, certaines approximations ont été introduites, dont l'influence n'était pas à même de modifier l'ordre de grandeur de la variation de quantité de mouvement.

\section{Forces extérieures}

- Forces de pressions hydrostatiques

$$
\bar{\omega}_{2}^{\bar{\omega} b}\left[h^{2}-(h-\Delta h)^{2}\right]+\frac{\bar{\omega} n}{3}\left[h^{3}-(h-\Delta h)^{3}\right]
$$

$\bar{\omega}=$ poids spécifique de l'eau

$h=$ profondeur du canal au repos

$b=$ largeur au plafond

$n=$ fruit des talus

- Forces de frottement sur le canal

$$
\rho C f_{c} \chi_{c} L_{b} \frac{v^{2}}{2}
$$

$C f_{c}=$ coefficient unitaire de frottement sur la surface mouillée du canal

$$
=\frac{2 g}{K_{c}^{2} R_{c}^{1 / 3}}
$$

$\chi_{c}=$ périmètre mouillé du canal

$L_{b}=$ longueur du bateau

$K_{c}=$ coefficient de Strickler du canal

$R_{c}=$ rayon hydraulique du canal

- Forces de frottement sur le bateau :

$$
\rho C f_{b} \chi_{b} L_{b} \frac{(V+v)^{2}}{2}
$$

avec

$C f_{b}=$ coefficient unitaire de frottement sur la surface mouillée longitudinale du bateau

$$
\begin{aligned}
& =\frac{\lambda_{b}}{4} \text { où } \lambda_{b}=\text { coefficient de perte de charge } \\
& \text { linéaire }=0,02
\end{aligned}
$$

$\chi_{b}=$ périmètre mouillé du bateau

- Forces de trainée :

$$
\rho C_{x} l_{b}(e+\Delta h) \frac{V^{2}}{2}
$$

avec

$C_{x}=$ coefficient de trainée $=1$

$e^{x}=$ enfoncement du bateau

$l_{b}=$ largeur du bateau

La résolution des équations 1 et 2 permet de déterminer $v$ et $\Delta h$.

Les pièces 1 et 2 donnent les abaissements obtenus sous forme de graphique en fonction de $V$, pour des canaux dont les pentes des talus sont respectivement $1 / 2$ et $1 / 3$.

\section{Etude sur modèle réduit physique}

Le modèle (voir photo) a été réalisé au laboratoire d'hydraulique de la Compagnie Nationale du Rhône à l'échelle du $1 / 50$.

La longueur du modèle (un peu plus de $30 \mathrm{~m}$ ) a permis l'implantation de deux sections différentes du canal, l'une avec un talus à $2 / 1$, l'autre avec un talus à $3 / 1$.

Les matériaux utilisés pour la réalisation des parois du canal, de la coque des berges et du pousseur ont été choisis de façon que leur rugosité corresponde exactement à celles des ouvrages réels, tenu compte de la similitude.

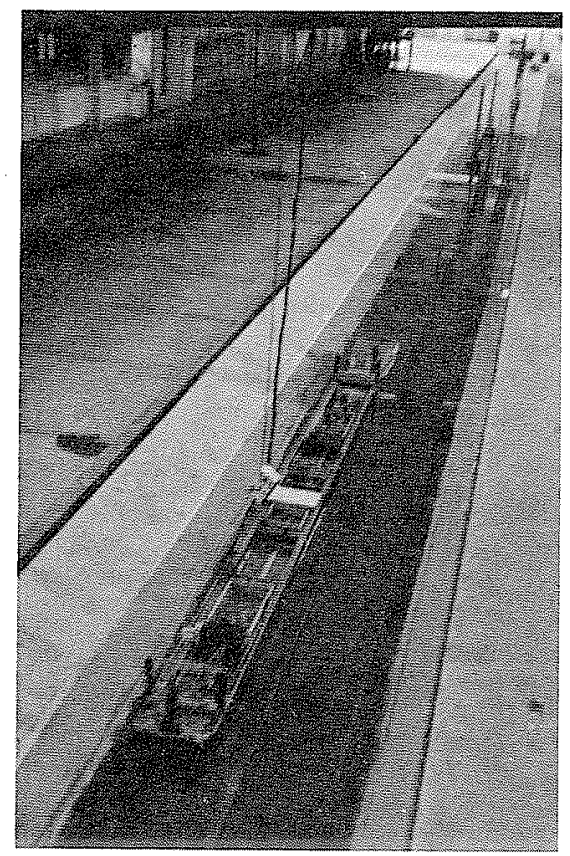



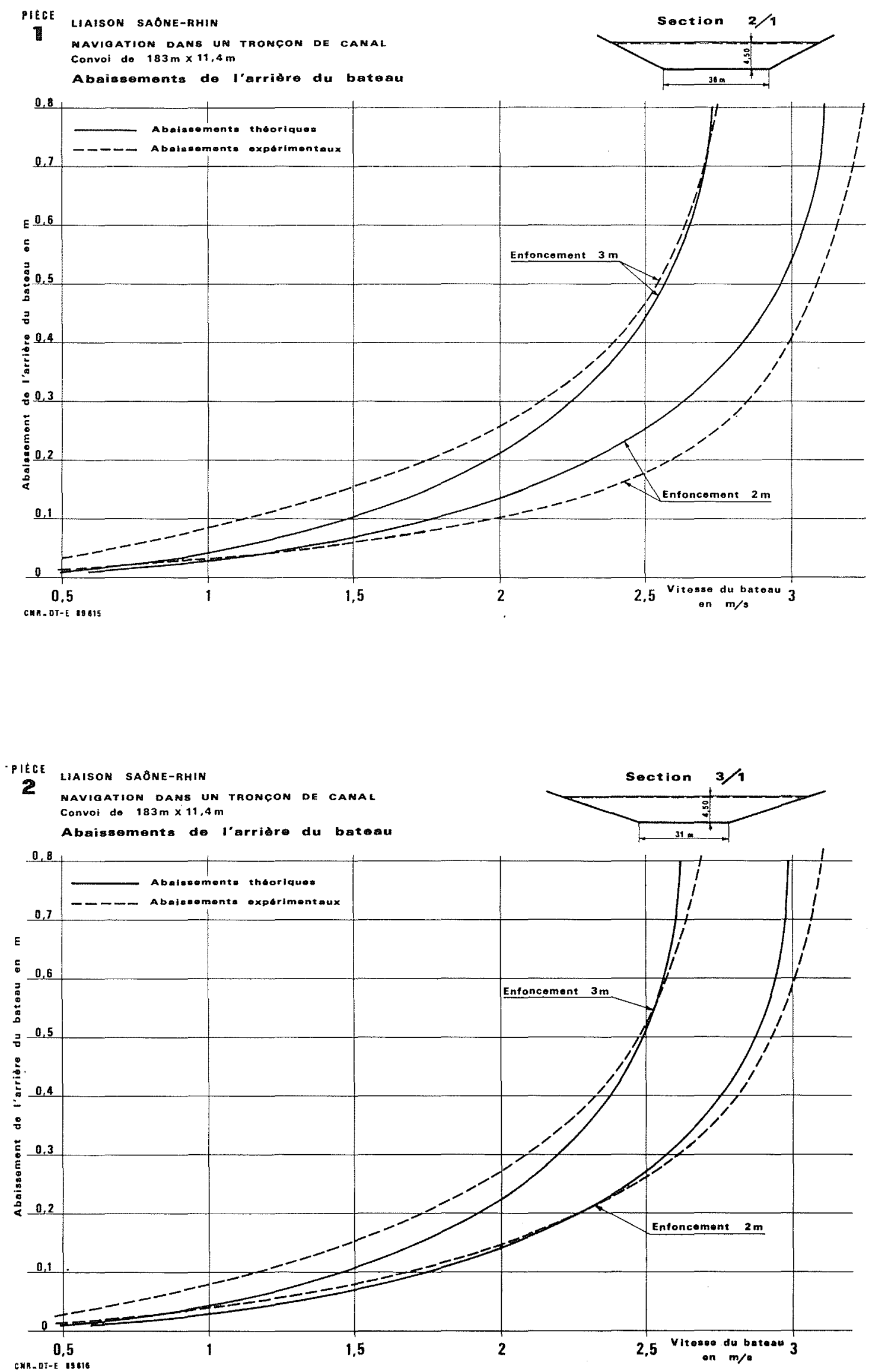
Des dispositions spéciales ont été prises en ce qui concerne l'alimentation en énergie électrique, la mesure de la vitesse du convoi, la mesure des pieds de pilote.

Les résultats ont été portés sur les courbes obtenues par modèle mathématique (pièces 1 et 2).

Les écarts que l'on peut observer n'étant pas significatifs, en particulier dans la zone des vitesses comprises entre $2 \mathrm{~m} / \mathrm{s}$ et $2,7 \mathrm{~m} / \mathrm{s}$, on peut considérer que les résultats du mođèle mathématique sont fiables ce qui permet de les extrapoler pour des canaux de formes et de dimensions diverses (pièce 3 ).

Toutefois, avant de passer à ces extrapolations, il convient d'examiner quels sont les efforts et puissances de poussage nécessaires pour déplacer le convoi à une vitesse donnée.

\section{Efforts et puissances de poussage}

\section{Etude théorique}

Il existe un certain nombre de formules pour déterminer la résistance à l'avancement des bateaux.

La formule de Gebers-Engels a été retenue; elle s'exprime sous une forme relativement simple :

$$
W=\left(K s+\lambda S_{m}\right)(V+v)^{2,25}
$$

$W \quad=$ résistance du bateau

$s \quad=$ maître couple de la coque immergée

$S_{m} \quad=$ surface mouillée de la coque

$V+v=$ vitesse relative du bateau

$K=$ coefficient dépendant de la forme de la coque et de l'enfoncement $\lambda=$ coefficient dépendant de la rugosité de la coque

Cette formule qui est actuellement la plus utilisée, fait intervenir la vitesse relative du convoi à l'exposant 2,25 . Elle n'est donc pas homogène et son application doit se faire avec prudence.

Pour vérifier si son domaine d'application était suffisamment large pour couvrir la gamme des vitesses utilisées par un convoi circulant dans un canal, ainsi que pour déterminer les coefficients $K$ et $\lambda$, il a été fait appel au modèle réduit.

\section{Etude sur modèle réduit}

L'échelle de base du modèle conduisant à une échelle de puissance très faible (1/883.883), de grandes précautions ont été prises pour l'enregistrement des efforts. Les sondes de mesure ont été placées entre les barges et le pousseur, elles ne mesuraient ainsi que la résistance à l'avancement des barges en négligeant celle du pousseur.

D'autre part, il a été tenu compte séparément de la résistance à l'avancement provoquée par le frottement du fluide sur les parois (forces de viscosité) et de la résistance induite par le système d'ondes liées au mouvement du convoi. La similitude de Froude n'a été appliquée qu'à cette seconde composante de la résistance.

Sous ces réserves, il a été constaté que la formule de Gebers-Engels permettait d'obtenir avec une bonne approximation les efforts de poussage, tenu compte des coefficients $K$ et $\lambda$ indiqués ci-après, et qu'à chaque

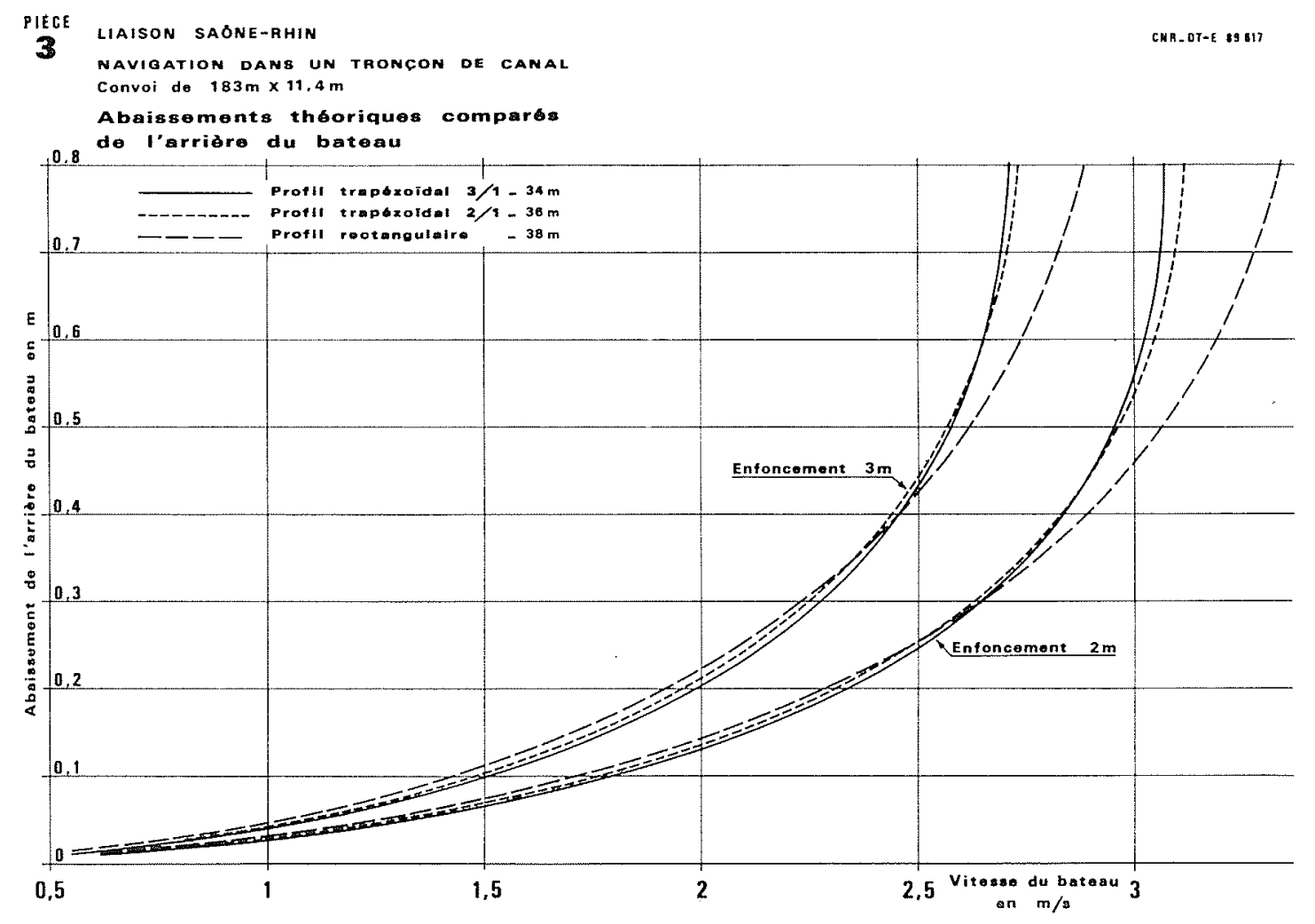




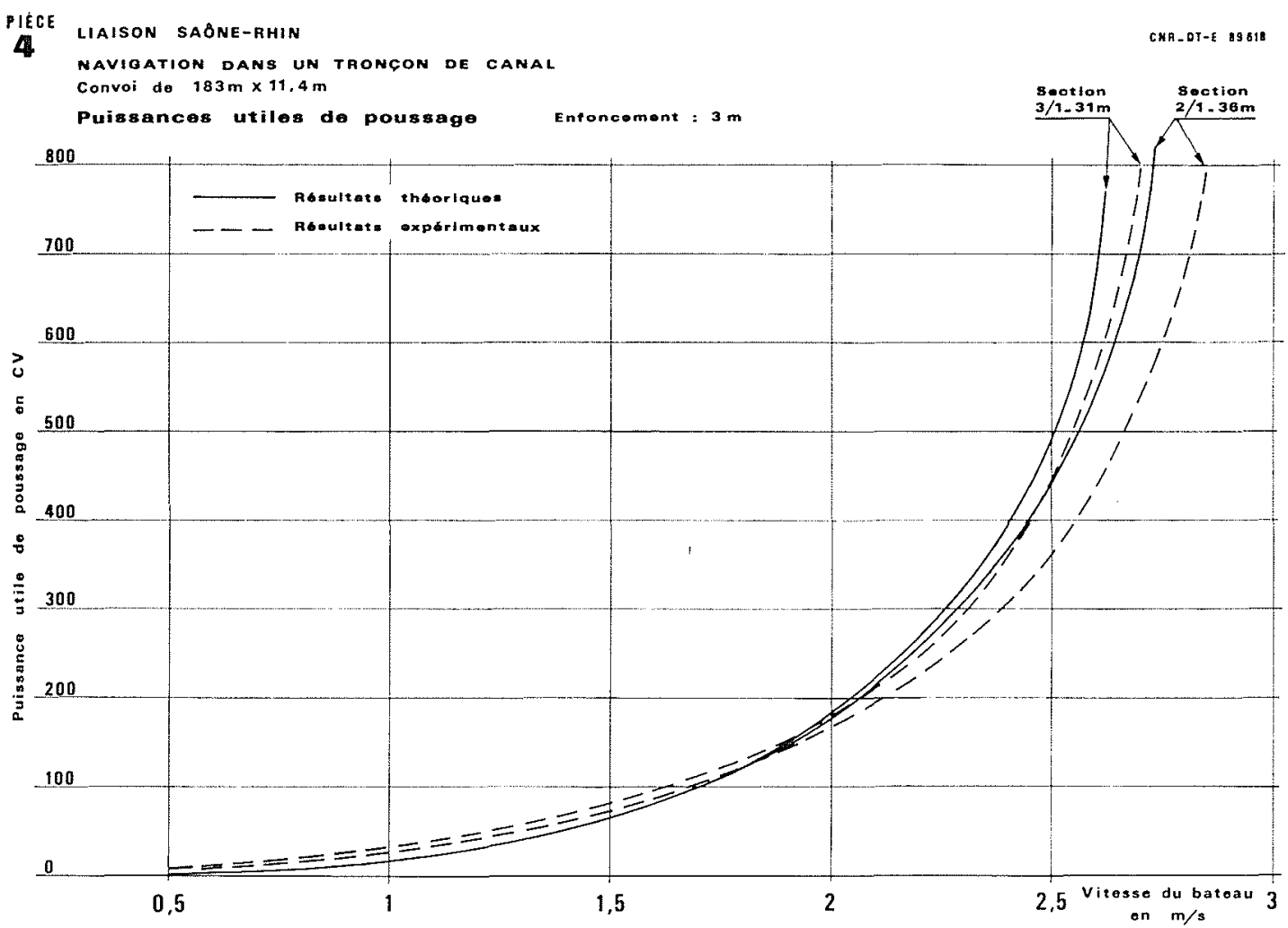

couple de valeurs $k, \lambda$ correspondait une répartition particulière de la puissance totale.

Il est à noter enfin que le coefficient $K$ varie légèrement avec la vitesse.

\begin{tabular}{|c|c|c|c|c|}
\hline enfoncement & $K$ & $\lambda$ & $\begin{array}{c}\text { maître } \\
\text { couple }(K)\end{array}$ & $\begin{array}{c}\text { résistance } \\
\text { coque }(\lambda)\end{array}$ \\
\hline $2 \mathrm{~m}$ & 3 & 0,19 & $11 \%$ & $89 \%$ \\
\hline $3 \mathrm{~m}$ & 5,8 & 0,19 & $25 \%$ & $75 \%$ \\
\hline
\end{tabular}

On mesure ainsi l'importance de la résistance liée à la coque, d'où l'obligation d'un bon entretien de celle-ci pour éviter l'accroissement des frais d'exploitation.

Les puissances utiles de poussage pour un enfoncement de $3 \mathrm{~m}$, correspondant à deux sections déterminées de canaux, sont données par le graphique, pièce 4 .

\section{Caractéristiques géométriques des canaux}

En définitive, ces études ont montré qu'il était possible de respecter des conditions fixées, à savoir :

- vitesse du convoi : $8,5 \mathrm{~km} / \mathrm{h}=2,36 \mathrm{~m} / \mathrm{s}$;

- puissance utile : $350 \mathrm{CV}$ environ;

avec des profils en travers de forme trapézoïdale (talus $\grave{a} 2 / 1$ et $3 / 1$ ) et rectangulaire. Les caractéristiques de ces profils qui tiennent également compte de conditions supplémentaires telles que largeur du canal, mouillage, vitesse du courant de retour $(0,75 \mathrm{~m} / \mathrm{s})$ sont données dans le tableau ci-après :

\begin{tabular}{|c|c|c|c|c|c|c|}
\hline \multirow{2}{*}{$\begin{array}{c}\text { Enfonce- } \\
\text { ment }(\mathrm{m}) \\
\text { Talus de berge }\end{array}$} & \multicolumn{3}{|c|}{$3 m$} & \multicolumn{3}{|c|}{$4 m$} \\
\hline & $\begin{array}{c}\text { Verti- } \\
\text { cal }\end{array}$ & $2 / 1$ & $3 / 1$ & $\begin{array}{c}\text { Verti- } \\
\text { cal }\end{array}$ & $2 / 1$ & $3 / 1$ \\
\hline $\begin{array}{l}\text { Largeur du } \\
\text { plan d'eau } \\
\text { (m) }\end{array}$ & 38 & 54 & 61 & 38 & 54 & 61 \\
\hline $\begin{array}{l}\text { Largeur au } \\
\text { plafond }(\mathrm{m})\end{array}$ & 38 & 36 & 34 & 38 & 31,20 & 26,80 \\
\hline Mouillage $(\mathrm{m})$ & 4,85 & 4,5 & 4,5 & 5,85 & 5,70 & 5,70 \\
\hline Section $\left(\mathrm{m}^{2}\right)$ & 184 & 202 & 214 & 222 & 243 & 250 \\
\hline $\begin{array}{l}\text { Rapport de } \\
\text { la section } \\
\text { mouillée au } \\
\text { maître couple }\end{array}$ & 5,4 & 5,9 & 6,3 & 4,9 & 5,3 & 5,5 \\
\hline
\end{tabular}

Il $\mathrm{y}$ a lieu de noter que ces profils permettent une vitesse des convois plus élevée dans l'hypothèse d'une marche des moteurs à $100 \%$ de leur puissance au lieu de $60 \%$. Il est alors possible d'atteindre $9,5 \mathrm{~km} / \mathrm{h}$. Toutefois, ce fonctionnement n'est pas économique car le gain de vitesse du convoi est de $12 \%$ alors que la puis. sance mobilisée est accrue de $70 \%$.

Enfin, on peut vérifier que la vitesse de base de $8,5 \mathrm{~km} / \mathrm{h}$ est égale à $85 \%$ de la vitesse limite ce qui est assez généralement reconnu comme la vitesse la plus performante.

En résumé, les profils ci-dessus définis, correspondent donc bien aux profils optimaux du point de vue économique. 


\section{Cas particuliers de la traversée de Mulhouse et du souterrain de Besancon}

Pour franchir certains passages difficiles, des profils réduits ont été prévus en particulier pour la traversée de Mulhouse et pour le souterrain de Besançon.

\section{Traversée de Mulhouse}

Dans la traversée de la ville de Mulhouse, la place disponible pour le passage du canal est limitée et la largeur maximale utilisable est de $31 \mathrm{~m}$. La réalisation d'un canal à berges verticales de cette largeur et d'une profondeur de $4,80 \mathrm{~m}$ permet le passage de convois à la vitesse de $6,8 \mathrm{~km} / \mathrm{h}$ avec une puissance utile de $180 \mathrm{CV}$ dans l'hypothèse où l'on se fixe de ne pas dépasser la vitesse du courant de retour retenu pour les profils normaux. Pour $350 \mathrm{CV}$ on arriverait à une vitesse de $8,1 \mathrm{~km} / \mathrm{h}$ mais le courant de retour attein drait $1,10 \mathrm{~m} / \mathrm{s}$ en moyenne et certainement davantage au voisinage du fond ce qui conduirait à prévoir une protection de celui-ci.

\section{Souterrain de Besançon}

Pour ce souterrain, il a été jugé suffisant de prévoir un ouvrage à voie unique avec alternance de circulation réglementée par des feux.

Tenu compte de la longueur du souterrain et de la présence en son extrémité de l'écluse de Besançon, il suffit que la moyenne des bateaux soit de l'ordre de
$5 \mathrm{~km} / \mathrm{h}$ pour que celui-ci n'affecte pas le débit de la voie.

La technologie des tunnels conduisant généralement à l'adoption de section en fer à cheval, une telle section a été retenue pour l'ouvrage de Besançon dont les dimensions sont les suivantes:

$$
\begin{array}{lc}
\text { - section totale } & 270 \mathrm{~m}^{2} \\
\text { - section mouillée } & 124 \mathrm{~m}^{2} \\
\text { - largeur du chenal } & 15 \mathrm{~m} \\
\text { - mouillage } & 6 \mathrm{~m}
\end{array}
$$

Elle permet au convoi de $3 \mathrm{~m}$ de mouillage une vitesse de $5 \mathrm{~km} / \mathrm{h}$ avec une puissance utile de $70 \mathrm{CV}$ et un abaissement du bateau de $0,15 \mathrm{~m}$. Ainsi, il reste une importante marge de sécurité puisqu'avec la puissance utile de $350 \mathrm{CV}$, la vitesse du bateau serait de $7,8 \mathrm{~km} / \mathrm{h}$ et l'abaissement du bateau de $0,50 \mathrm{~m}$ environ.

Le courant de retour dans ce dernier cas serait assez élevé puisqu'il atteindrait $1,20 \mathrm{~m} / \mathrm{s}$ en moyenne, contre $0,6 \mathrm{~m} / \mathrm{s}$ pour la vitesse de $5 \mathrm{~km} / \mathrm{h}$, mais ceci est sans inconvénient puisque le tunnel est revêtu.

\section{Conclusion}

Les calculs théoriques bien que basés sur des hypothèses simplificatrices indispensables du fait de la complexité des phénomènes, conduisent à des résultats qui représentent convenablement l'ordre de grandeur des différentes variables liées au déplacement du bateau.

Ils permettent de définir les caractéristiques des profils en travers types d'une voie navigable homogène. 